\title{
CNS myeloid cells critically regulate heat hyperalgesia
}

\author{
Stefanie Kälin, ${ }^{1}$ Kelly R. Miller, ${ }^{1}$ Roland E. Kälin, ${ }^{1}$ Marina Jendrach,, ${ }^{1}$ Christian Witzel, ${ }^{2}$ and Frank L. Heppner ${ }^{1,3,4,5}$ \\ 'Department of Neuropathology and '2Department of Plastic Surgery, Charité - Universitätsmedizin Berlin, Corporate Member of Freie Universität Berlin, Humboldt-Universität zu Berlin, and Berlin Institute \\ of Health, Berlin, Cermany. ${ }^{3}$ Cluster of Excellence, NeuroCure, Berlin, Germany. ${ }^{4}$ Berlin Institute of Health, Berlin, Germany. ${ }^{5}$ Cerman Center for Neurodegenerative Diseases (DZNE) Berlin, Berlin, Cermany.
}

\begin{abstract}
Activation of non-neuronal microglia is thought to play a causal role in spinal processing of neuropathic pain. To specifically investigate microglia-mediated effects in a model of neuropathic pain and overcome the methodological limitations of previous approaches exploring microglia function upon nerve injury, we selectively ablated resident microglia by intracerebroventricular ganciclovir infusion into male CD11b-HSVTK-transgenic mice, which was followed by a rapid, complete, and persistent ( 23 weeks) repopulation of the CNS by peripheral myeloid cells. In repopulated mice that underwent sciatic nerve injury, we observed a normal response to mechanical stimuli, but an absence of thermal hypersensitivity ipsilateral to the injured nerve. Furthermore, we found that neuronal expression of calcitonin gene-related peptide (CGRP), which is a marker of neurons essential for heat responses, was diminished in the dorsal horn of the spinal cord in repopulated mice. These findings identify distinct mechanisms for heat and mechanical hypersensitivity and highlight a crucial contribution of CNS myeloid cells in the facilitation of noxious heat.
\end{abstract}

\section{Introduction}

Neuropathic pain refers to a complex chronic pain state resulting from damage to or dysfunction of the somatosensory system characterized by mechanical allodynia, hyperalgesia, and spontaneous pain in human patients. There is accumulating evidence that neurons are not the only crucial players in spinal processing of nociceptive signals, but that glial cells also contribute to experimental pain states. In particular, microglia, the resident immune cells in the CNS, are powerful modulators in the induction of nociception $(1,2)$. As a consequence of peripheral nerve injury, microglia in the dorsal horn $(\mathrm{DH})$ of the spinal cord rapidly respond to injury by migrating to the site of damage, proliferating, upregulating a variety of cellsurface receptors, and elaborating an array of cytokines. The latter can be causal for the hyperactivity of nociceptive neurons. In addition to the activation of CNS-resident microglia, peripheral axonal injury also results in the recruitment of hematogenous monocytes to the $\mathrm{DH}$, where these monocytes also provide a rich source of pain mediators that act on nociceptive terminals (3).

The necessity of microglia and peripheral blood-borne myeloid cells for the initiation of neuropathic pain processing after peripheral nerve injury has not been independently investigated to date, given the lack of appropriate tools to address this question. Thus, we used a transgenic mouse model that allows for large-scale depletion of microglia in the CNS, namely, mice expressing the CD11b promoter-driven herpes simplex virus thymidine kinase (CD11b-HSVTK, referred to hereafter as TK mice) $(4,5)$. Following microglia ablation in this model, peripheral monocytic cells rapidly infiltrate and repopulate the brain parenchyma (6), thus allowing for the effective exchange of

Authorship note: SK and RKM contributed equally to this work. Conflict of interest: The authors have declared that no conflict of interest exists. Submitted: May 24, 2017; Accepted: March 27, 2018.

Reference information: J Clin Invest. 2018;128(7):2774-2786.

https://doi.org/10.1172/JCI95305. endogenous microglia with peripheral myeloid cells (7). Using the TK mouse model, we found that, following partial sciatic nerve ligation (PSNL), mice lacking central microglia that were replaced with peripheral myeloid cells failed to develop heat hypersensitivity, but maintained normal responses to mechanical and cold stimulation. In line with this finding, we observed a substantial reduction in CGRP, a molecular marker of peptidergic nociceptive neurons that were shown to be required to sense heat (8). Taken together, we report for the first time to our knowledge on an animal model allowing rapid recruitment of peripheral myeloid cells into the spinal cord parenchyma after depletion of resident microglia. In this way, we were able to experimentally show that the origin of myeloid cells, determines, at least to some extent, their functional repertoire, demonstrating that the role of resident microglia in neuropathic pain is distinct from that of peripheral myeloid cells and extends beyond acute proinflammatory responses that promote the development of pain, but also encompasses the mediation of distinct pain entities.

\section{Results}

Myeloid cell response to peripheral nerve injury. Microglial activation can be consistently observed in a variety of experimental peripheral nerve injury models $(1,9)$. Induction of glial cytokine expression is aligned with morphological changes and increased expression of the myeloid cell-specific marker Iba1 (10). To gain a better understanding of the temporal activation pattern of microglia after PSNL in WT mice, we analyzed Iba1 immunoreactivity (IR) in the ipsilateral and contralateral dorsal horn (DHi/ $\mathrm{DHc}$ ) of the lumbar spinal cord at several time points after PSNL. Compared with the contralateral side, microglia surrounding the injured sensory and motor neurons of the sciatic nerve terminals in the DHi and ipsilateral ventral horn (VHi) showed intense IR (Figure 1A). In the DHi, quantitative morphometric analysis of the area covered by $\mathrm{Iba}^{+}$cells revealed a substantial increase 
A

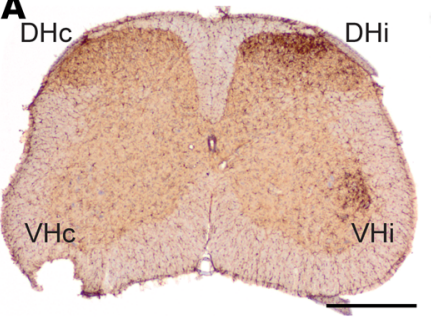

Iba1; 7 dpi

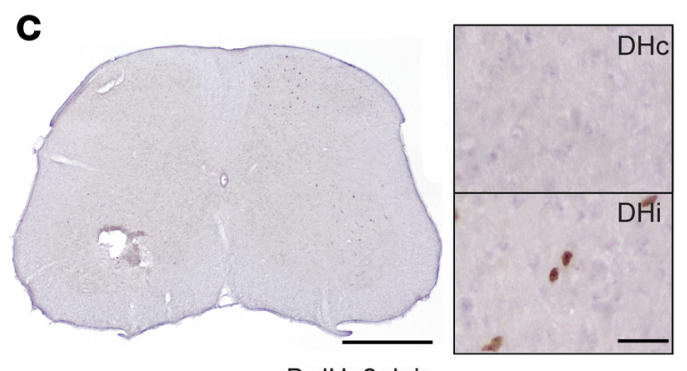

BrdU; 2 dpi

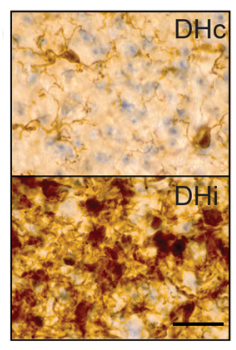

B

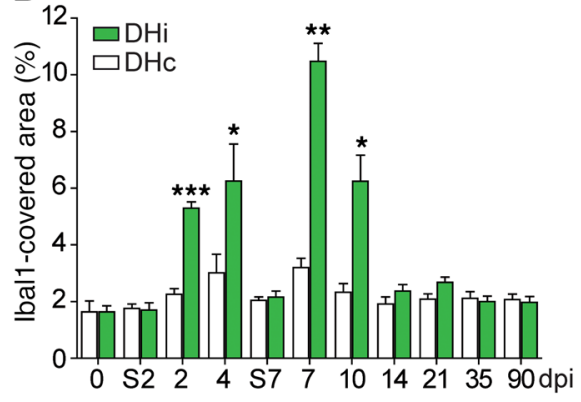

D

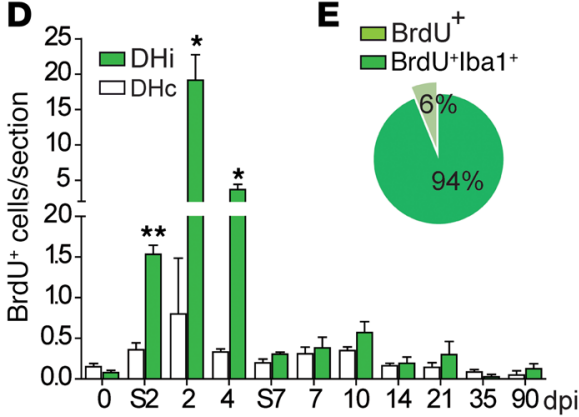

$\mathbf{F}$

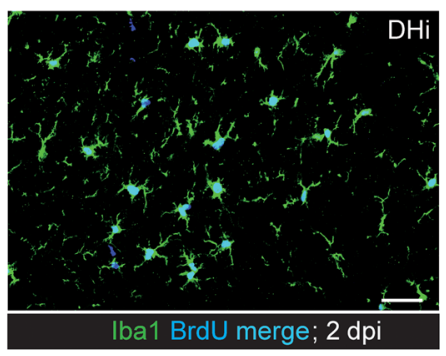

G

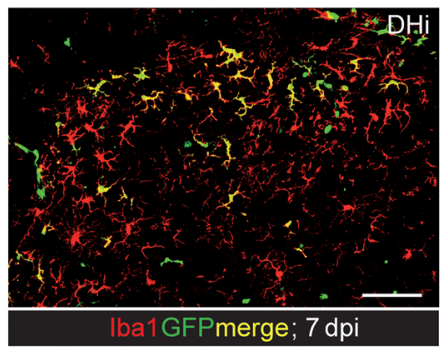

Figure 1. PSNL evokes reactive microgliosis and infiltration of bone marrow-derived myeloid cells into the DHi of WT mice. (A) An increase in Iba1-IR in the lumbar spinal cord of WT animals was observed in the DHi and VHi. Scale bars: $500 \mu \mathrm{m}$ and $25 \mu \mathrm{m}$ (insets). (B) The temporal profile of Iba1 ${ }^{+}$microglia activation was determined in the $\mathrm{DHi}$ and $\mathrm{DHc}$ of the lumbar spinal cord (8-10 sections/mouse, 4 mice/time point) and revealed a significant increase in the Iba1-covered area from 2 to $10 \mathrm{dpi}$, peaking at $7 \mathrm{dpi}$. (C) Increased numbers of BrdU+ cells were detected in the lumbar DHi and VHi. Scale bars: $500 \mu \mathrm{m}$ and 25 $\mu \mathrm{m}$ (insets). (D) Within the $\mathrm{DHi}$, the highest number of BrdU+ cells was found at 2 and $4 \mathrm{dpi}$ (8-10 sections/mouse, 4 mice/time point). (E) Pie chart shows that $94 \%$ of total BrdU+ cells were also lba1+. (F) PSNL-induced proliferation of Iba1 (green) and BrdU (blue) double-positive cells (merge) was restricted to the DHi. Scale bar: $25 \mu \mathrm{m}$. (G) Confocal microscopy revealed bone marrow-derived GFP+ cells (green) colocalizing with the myeloid cell marker Iba1 (red) in the DHi of GFP-bone marrow chimeric mice on day 7 after PSNL. Scale bar: $50 \mu \mathrm{m}$. Error bars indicate the SEM. ${ }^{*} P<0.05,{ }^{* *} P<0.01$, and ${ }^{* * *} P<0.001$, by paired, 2-tailed Student's $t$ test for comparison of DHc and DHi at each time point. S, sham.

starting 2 days post injury (dpi), peaking at 7 dpi, and declining thereafter (Figure 1B).

We assessed the proliferation of microglia by BrdU incorporation at multiple time points after injury. Compared with naive lumbar spinal cords, in which only a few sparse BrdU-immunolabeled cells could be found within the spinal cord parenchyma, we detected vast numbers of newly dividing BrdU+ cells within the DHi from 2 to 4 dpi (Figure 1, C and D). Double immunofluorescence staining of incorporated BrdU and Iba1 (Figure $1 \mathrm{~F}$ ) revealed that $94 \%$ of BrdU $^{+}$cells in the DHi were $\mathrm{Iba1}^{+}$(Figure 1E).

To unequivocally distinguish blood-derived myeloid cells from intrinsic microglia, we generated chimeric mice harboring isogenic $\beta$-actin-GFP-labeled WT bone marrow. Double immunolabeling revealed a clear colocalization of GFP and Iba1 (Figure 1G), confirming that, in addition to resident microglia, peripheral myeloid cells also contributed a minor amount to the $\mathrm{Iba}^{+}$cell population within the lumbar spinal cord in the early activation phase after PSNL.

Depletion of microglia and persistent repopulation with peripheral myeloid cells in the lumbar spinal cord. Circulating monocytes do not substantially enter or engraft the CNS of healthy mice (11); however, specific pathological conditions, such as peripheral nerve injury, trigger their infiltration $(3,12)$. To investigate whether behavioral differences in the facilitation of pain signals exist between CNSresident microglia and peripheral myeloid cells, we took advantage of the TK-transgenic mouse model, which allows for the central depletion of endogenous $\mathrm{CD}_{11 \mathrm{~b}^{+}}$microglia in the brain parenchy- ma, followed by rapid repopulation of peripheral myeloid cells upon intracerebroventricular (i.c.v.) administration of the drug ganciclovir (GCV) $(6,7)$. However, prior to this study, it remained unclear whether other parts of the CNS, namely the lumbar spinal cord, can also be repopulated with peripheral myeloid cells and whether they can functionally replace CNS-resident microglia. Thus, a specific exchange protocol for the spinal cord was established that takes advantage of the rapid transport of GCV via the cerebrospinal fluid (CSF) to the lumbar spinal cord. To restrict GCV sensitivity to resident microglia and distinguish between remaining microglia and peripheral myeloid cells after CNS repopulation, we generated GFP bone marrow chimeric mice that only express the TK transgene in the radioresistant $\mathrm{CNS}$ (GFP>TK), as well as nontransgenic WT littermates (GFP>WT).

To circumvent potential side effects of high CCL2 expression, which has been reported to be produced upon irradiation and involved in the recruitment of CCR2-expressing myeloid cell into the CNS (13), we waited 8 weeks after irradiation and reconstitution with GFP bone marrow before performing further manipulations (12). Two weeks after initiation of GCV treatment, quantitative stereological analysis revealed that $75 \%$ of the myeloid cell pool in the lumbar spinal cord of GFP $>$ TK animals was composed of $\mathrm{GFP}^{+}$peripherally derived cells (Figure $2 \mathrm{~B}$ ). GFP $>\mathrm{TK}$ mice that were analyzed 7 weeks (short term) after termination of GCV treatment had $92 \%$ repopulation (Figure 2, A and C). For all time points tested, GCV-treated GFP $>$ WT mice (Figure 2, B and C), 

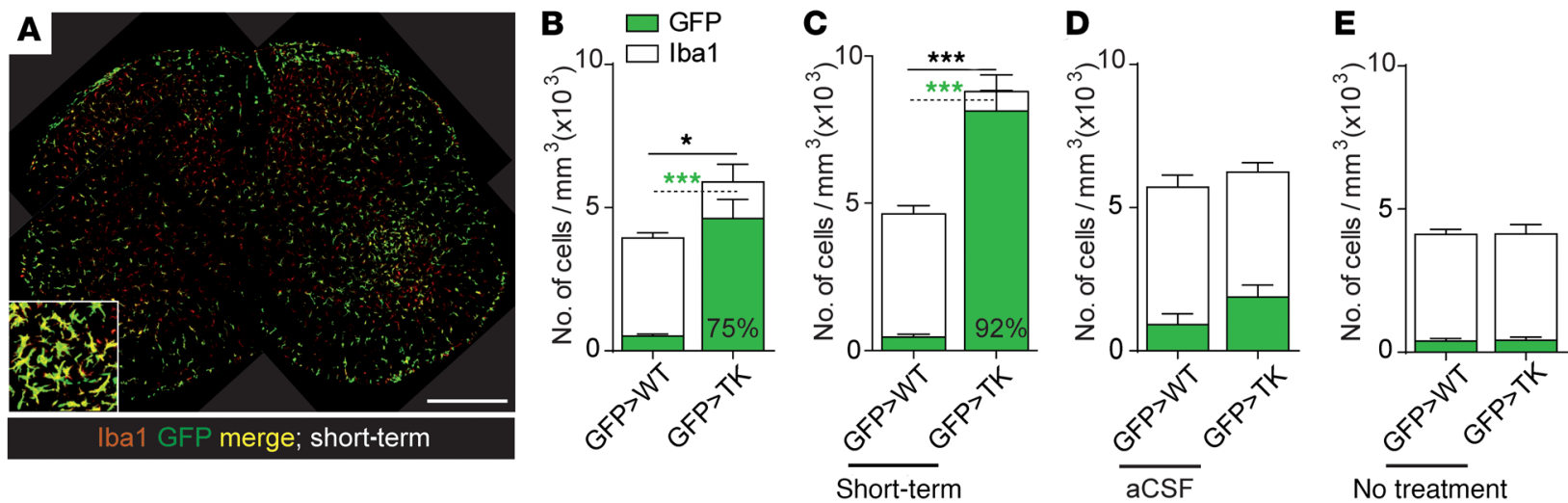

Figure 2. Repopulation in GFP>TK animals. (A) Confocal microscopic analysis (merged image) of peripherally derived myeloid cells in the lumbar spinal cord revealed that almost all GFP+ cells (green) were also lba1+ (red) after microglia depletion. Scale bar: $500 \mu \mathrm{m}$. Inset, original magnification, $\times 40$. (B and C) Quantitative stereological analysis of total Iba1 ${ }^{+}$and GFP+ cells in the contralateral lumbar spinal cord of GFP>TK mice treated with GCV, either continuously $(n=8)$ or short term $(n=10)$, revealed a $75 \%$ and $92 \%$ repopulation with peripheral myeloid cells, respectively, whereas their corresponding GFP>WT littermates (continuous GCV treatment, $n=10$; short-term GCV treatment, $n=9$ ) showed an average of only $10 \%$ GFP' cells. ( $(D$ and $\mathbf{E})$ Vehicle-treated (aCSF-treated) ( $n=8$ /genotype) as well as nontreated GFP>WT $(n=9)$ and GFP>TK $(n=4)$ mice showed only moderate infiltration of peripheral myeloid cells. The dashed line and green asterisks are shown for comparison of GFP+ cells. Error bars indicate the SEM. ${ }^{*} P<0.05$ and ${ }^{* * *} P<0.001$, by paired, 2-tailed Student's $t$ test for corresponding GFP>WT and GFP>TK pairs.

vehicle-treated mice (artificial CSF [aCSF]; Figure 2D), as well as nontreated GFP $>$ WT and GFP $>$ TK mice (Figure 2E) showed little to no infiltration of $\mathrm{GFP}^{+}$myeloid cells into the lumbar spinal cord, indicating that irradiation, reconstitution, and GCV administration, per se, did not promote a substantial invasion of peripheral myeloid cells. Notably, the number of $\mathrm{Iba1}^{+}\left(\right.$and GFP ${ }^{+}$) cells increased over time in the spinal cord tissue of GCV-treated GFP $>$ TK mice to an extent similar to that observed in repopulated brain regions $(6,7)$.

Interestingly, we observed long-term residency of peripherally derived $\mathrm{GFP}^{+}$myeloid cells in the lumbar spinal cord, even half a year after microglia depletion. Specifically, GFP $>$ TK mice that were analyzed 23 weeks (long term) after termination of GCV treatment exhibited $83 \%$ repopulation (Figure 3A). Moreover, analysis of the mean distance between $\mathrm{Iba} 1^{+}$cells revealed a denser distribution of repopulated cells in GFP $>$ TK mice than of resident microglia in the lumbar spinal cord of GFP $>$ WT animals (Figure 3, B and C).

Taken together, peripheral myeloid cell repopulation is dependent on microglia depletion in GFP $>$ TK mice. Compared with resident microglia, we found, as expected, that infiltrating myeloid cells were more numerous throughout the spinal cord, since they are tasked with covering the same surveillance area as microglia but lack the elaborate branched processes possessed by CNSresident microglia.

Long-term myeloid cell accumulation and activation in the DHi after PSNL in repopulated GFP $>T K$ mice. We observed reactive microgliosis at early time points (7 dpi) after PSNL in WT (Figure $1 \mathrm{~A}$ ), GFP $>\mathrm{WT}$, and GFP $>\mathrm{TK}$ (Figure $4 \mathrm{~A}$ ) mice. To compare the activation kinetics of engrafted myeloid cells with that of resident microglia, we performed immunohistological analyses of the DH of nonrepopulated GFP $>$ WT and repopulated GFP $>$ TK animals at late time points (50 dpi) after PSNL. We found no myeloid cell accumulation and little infiltration of $\mathrm{GFP}^{+}$peripheral cells in the uninjured (Contra) and injured (Ipsi) DH of GCV-treated
GFP $>$ WT animals (Figure 4, G and H) and nontreated (Figure 4B) or aCSF-treated GFP $>$ WT and GFP $>$ TK animals (Figure 4C) at any of the time points examined. As expected, repopulated sham-operated GFP $>$ TK animals showed no signs of myeloid cell accumulation (Figure 4, D and E). Instead, we identified a robust accumulation of $\mathrm{Iba}^{+} \mathrm{GFP}^{+}$myeloid cells that were still hypertrophic (Figure 4F), indicating an enhanced response state at $50 \mathrm{dpi}$ in the DHi of GFP $>$ TK animals, as compared with WT animals at 7 dpi. This observation was made in 2 independent experiments, namely in the short-term group (Figure 4, G and $\mathrm{H}$ ), in which PSNL was performed 2 weeks after depletion and repopulation took place, and in the long-term group (Figure 4, I and J), in which there was a period of 4 months between the time of GCV administration (and microglia depletion/myeloid cell repopulation) and PSNL, thus allowing the invading peripheral myeloid cells to adapt to the CNS environment.

Taken together, in contrast to resident microglia in WT and GFP $>$ WT mice, which returned to a homeostatic state 1 week after PSNL, infiltrating myeloid cells continued to exhibit a specific reactive phenotype in response to PSNL beyond 50 dpi, suggesting a differential response of endogenous microglia and peripherally derived CNS myeloid cells to PSNL at late time points.

Microglia-depleted and myeloid cell-repopulated mice lack heat hyperalgesia but react normally to mechanical and cold stimuli in response to PSNL. Injury to a peripheral nerve produces profound behavioral indicators of persistent pain, including hyperalgesia and allodynia (14). In WT mice, PSNL had no influence on motor function or reflexes, as assessed by the accelerating RotaRod and tail-flick tests, respectively; however, PSNL produced classic neuropathic pain symptoms, including a decrease in thermal and mechanical paw withdrawal thresholds (PWTs) ipsilateral to the injury (Supplemental Figure 1; supplemental material available online with this article; https://doi.org/10.1172/JCI95305DS1).

To specifically test whether peripheral myeloid cells are able to functionally replace microglia, we performed PSNL (or a sham 
A

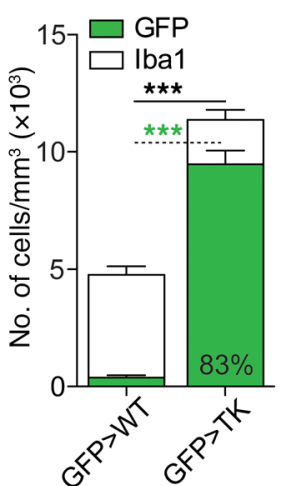

B

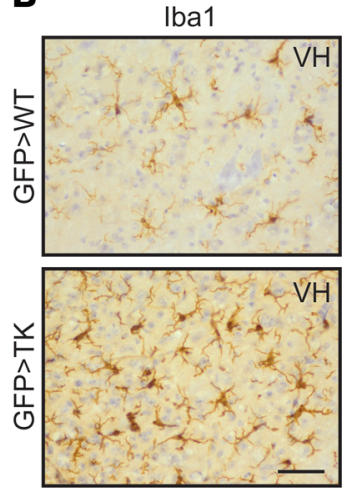

C

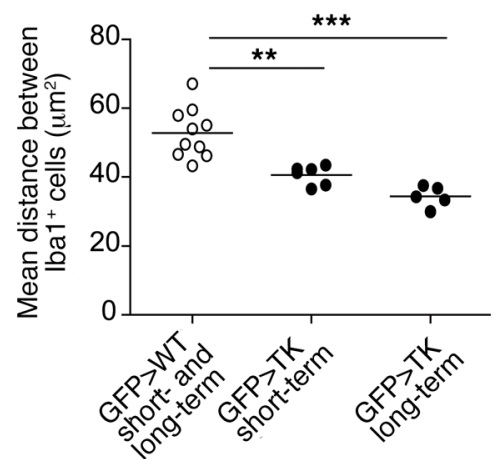

Figure 3. Sustained repopulation in GFP>TK animals. (A) In GFP>TK mice $(n=5), 83 \%$ of all myeloid cells in the lumbar spinal cord were of peripheral origin (GFP+) 6 months after termination of GCV treatment, while no repopulation was observed in GFP>WT mice $(n=5)$. (B and $\mathbf{C})$ Infiltrating myeloid cells were more numerous in GFP $>$ TK mice ( $n=5$ short-term, $n=5$ long-term) than in GFP>WT control mice $(n=10)$, resulting in a higher cell density than that of resident microglia. Scale bar: $50 \mu \mathrm{m}$. The dashed line and green asterisks are shown for comparison of GFP+ cells. Error bars indicate the SEM. ${ }^{*} P<0.01$ and ${ }^{* *} P<0.001$, by 1 -way ANOVA with Bonferroni's post hoc analysis. months after GCV treatment and microglia depletion and myeloid cell engraftment of peripheral myeloid cells (long term) confirmed this observation, as these mice also failed to manifest heat hyperalgesia (Figure 5I), but displayed normal mechanical responses (Figure $5 \mathrm{H}$ ). On the other hand, GFP $>$ WT control mice treated long term showed the expected mechanical and heat hyperalgesia (Figure 5, H and I). Again, neither group had alterations in the RotaRod or tail-flick test (Figure 5, F and G).

Collectively, in chronic pain tests, GFP $>$ TK animals depleted of central microglia and populated with bone marrowderived myeloid cells showed considerably decreased PWTs in response to mechanical and cold, but not heat, stimuli ipsilateral to the injury.

Microglia depletion and myeloid cell repopulation does not influence established operation) 2 weeks after continuous GCV treatment. At that time point, the lumbar spinal cord was already repopulated by peripherally derived $\mathrm{GFP}^{+}$cells (Figure 2B). To determine whether behavioral responses to heat, cold, and mechanical stimuli were affected by the lack of endogenous microglia and presence of peripherally derived myeloid cells, the mice were subjected to plantar, incremental cold plate, and von Frey tests, respectively. For all experiments, although GCV administration was stopped prior to PSNL, we assessed GCV- or aCSF-treated GFP>WT and GFP>TK mice before and after GCV or aCSF administration to exclude nonspecific effects of surgical procedures and prior GCV treatment. Untreated GFP $>$ WT and GFP $>$ TK animals had similar baseline thresholds for mechanical and thermal stimuli. In all groups tested, GCV treatment did not produce any baseline changes in PWTs in any of the behavioral tests (Figure 5, A-E), indicating that irradiation, reconstitution, and microglia depletion and myeloid cell repopulation had no effect on behavioral responses. Furthermore, we found that motor function (Figure 5A) and reflexes (Figure 5B) were not altered before or after PSNL in any of the tested groups.

In contrast, following PSNL, we observed mechanical allodynia and heat hyperalgesia in GCV-treated GFP $>$ WT and aCSF-treated GFP $>$ WT and GFP $>$ TK control animals. Shamoperated, repopulated GFP $>$ TK mice developed no behavioral hyperalgesia (Figure 5, C and D). Surprisingly, however, in GCV-treated GFP>TK mice, in which CNS-resident microglia were depleted and had been replaced by peripherally derived myeloid cells, PSNL resulted in a lasting formation of mechanical allodynia (Figure 5C), while heat withdrawal latencies for the injured paw were identical to those of the uninjured contralateral side (Figure 5D), indicating a complete lack of heat hyperalgesia in the repopulated mice. To determine whether this phenotype is specific for heat, we also measured cold sensitivity in GFP $>$ WT and GFP $>$ TK mice. GFP $>$ WT and GFP $>$ TK mice showed areater sensitivity to cold after PSNL (Figure 5E), suggesting that microglia ablation and myeloid cell repopulation had no effect on cold hyperalgesia. Similar analyses in animals that received PSNL 4 chronic pain symptoms. To test whether ablation of microglia and subsequent repopulation with peripheral myeloid cells can rescue thermal hyperalgesia that occurs after peripheral nerve injury, GCV was administered $7 \mathrm{dpi}$, a time point at which robust thermal hyperalgesia and mechanical allodynia were already established (Figure 6 and Supplemental Figure 1). In contrast to our observations in previous experiments, initiation of microglia depletion and myeloid cell repopulation within the lumbar spinal cord of GCV-treated GFP>TK mice after PSNL did not result in any difference in mechanical or thermal thresholds compared with nondepleted and nonrepopulated nerve-injured GFP $>$ WT mice (Figure 6, C and D). We observed no deficits in the RotaRod (Figure 6A) or tail-flick tests (Figure 6B) in these mice.

This observation suggests that the influence of microglia and/ or peripheral macrophages on thermal hyperalgesia, or the lack thereof, as observed in the presence of peripheral myeloid cells, results from a lack of initiation at acute stages rather than an inhibition of maintenance of hyperalgesia.

CGRP expression is reduced in the DH of microglia-depleted mice. Increased excitatory synaptic transmission and decreased inhibitory synaptic transmission in the $\mathrm{DH}$ of the spinal cord are the two most important characteristics for the development of central sensitization $(15,16)$. To compare the transcription pattern of resident microglia and infiltrating myeloid cells, a quantitative PCRbased (qPCR-based) microarray (mouse pain: neuropathic and inflammatory) of all cell populations within the DH of microgliadepleted and myeloid cell-repopulated mice and identically treated controls was performed 7 dpi. Gene expression analysis led to the identification of a number of presumably microglia- and macrophage-specific inflammatory markers that were significantly upregulated in repopulated GFP $>$ TK mice (Supplemental Table 1). Yet, none of these target genes was found to be specifically regulated in the DHi compared with DHc, pointing toward a general inflammatory signature of bone marrow-derived myeloid cells repopulating the $\mathrm{CNS}$ after microglia depletion, rather than a specific response to PSNL. 
A
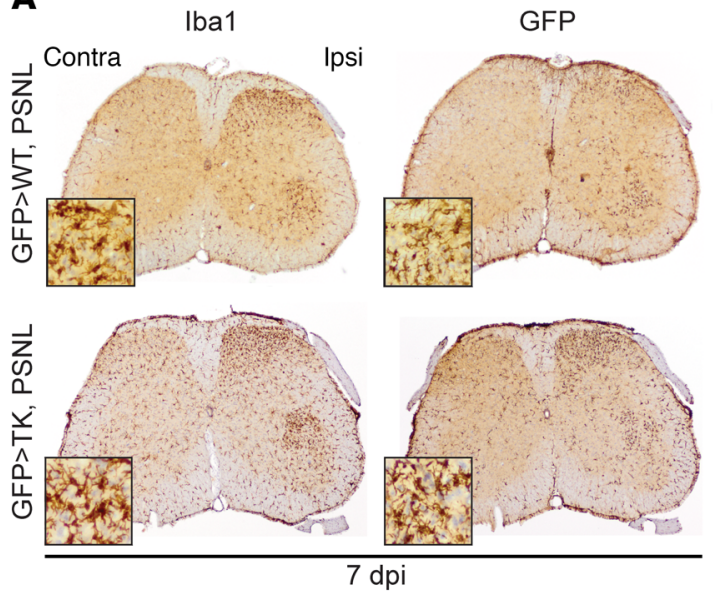

D

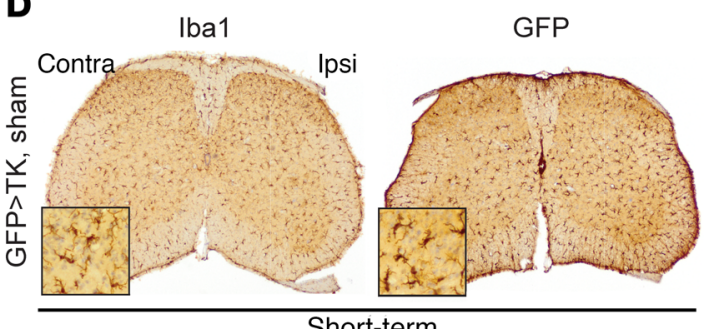

G
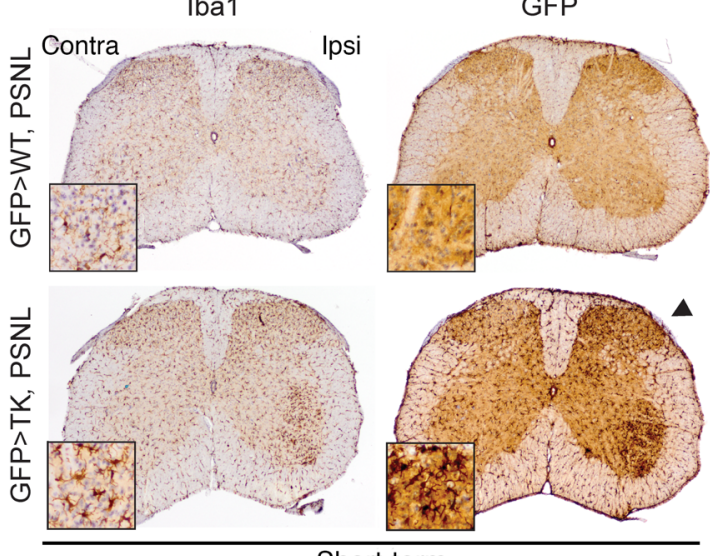

Short-term
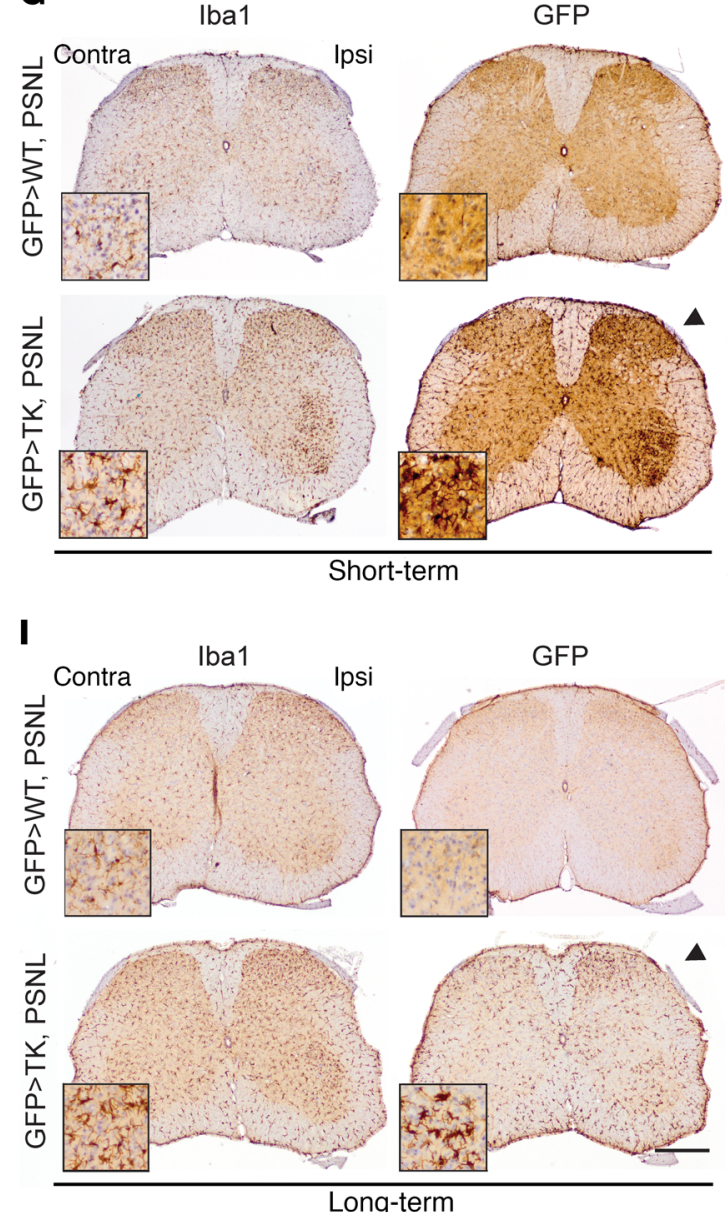

B

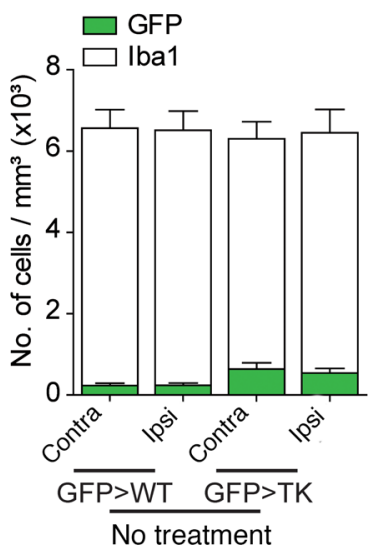

C

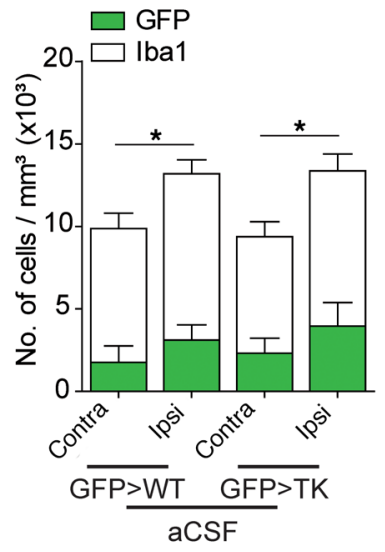

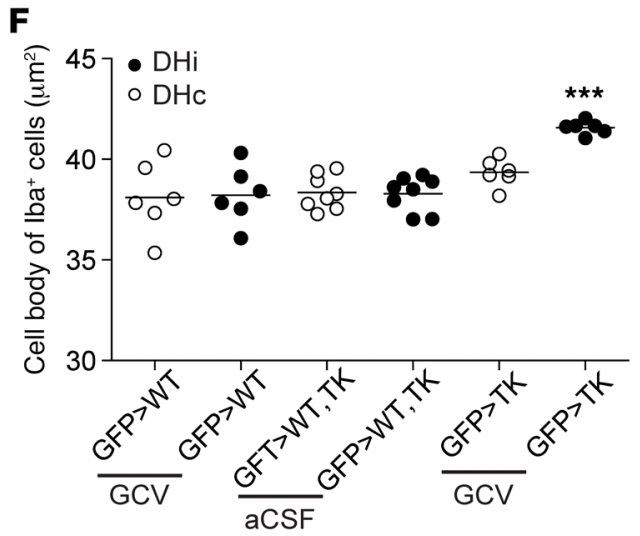

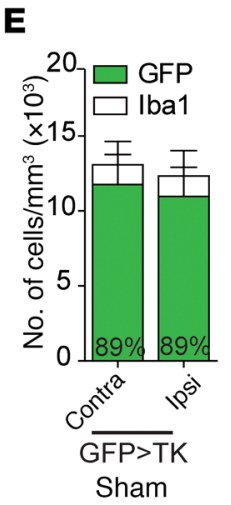

H

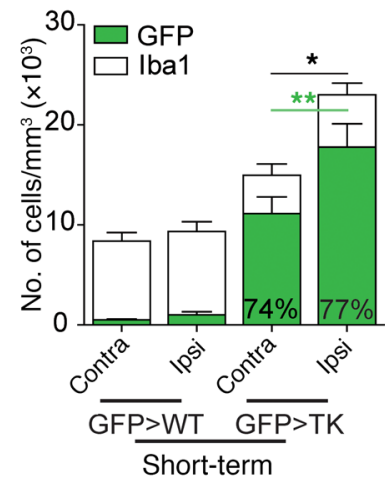

J

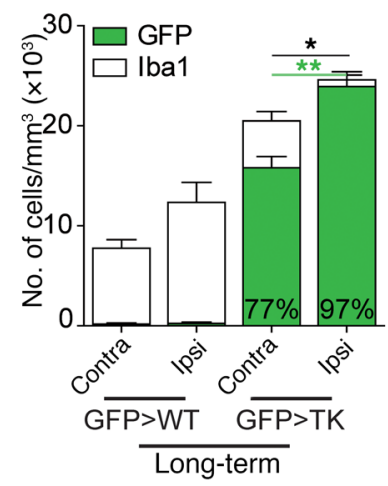


Figure 4. Protracted peripheral myeloid cell reactivity after PSNL in GFP>TK animals. (A) A marked increase in Iba1 and GFP-IR in the lumbar spinal cord in GFP $>$ WT and GFP $>$ TK animals was observed in the DHi and $\mathrm{VHi}$ at $7 \mathrm{dpi}$. (B) Stereological analysis of $\mathrm{Iba}^{+}$and $\mathrm{GFP}^{+}$cells in the $\mathrm{DHi}$ (Ipsi) or DHc (Contra) revealed no difference in the number of $\mathrm{Iba}^{+}$ or GFP+ cells in nontreated GFP $>$ WT $(n=6)$ and GFP>TK $(n=4)$ mice. (C) aCSF-treated GFP $>$ WT $(n=7)$ and GFP $>$ TK $(n=8)$ mice showed only moderate infiltration of peripheral myeloid cells, but a significantly higher number of $\mathrm{Iba}^{+}{ }^{+}$cells within the $\mathrm{DHi}$ at $50 \mathrm{dpi}$, suggesting long-term survival of microglia that proliferated early after PSNL. (D and E) At $50 \mathrm{dpi}, 89 \%$ of the cells in both the $\mathrm{DHi}$ and $\mathrm{DHc}$ were repopulated cells in sham-operated GFP>TK $(n=5)$ mice. (F) Infiltrating myeloid cells in the DHi of GCV-treated GFP $>$ TK mice had significantly larger cell bodies (combined short-term and long-term repopulation groups). (G) Lumbar spinal cord sections at $50 \mathrm{dpi}$ revealed increased numbers of GFP+ myeloid cells in GFP $>$ TK animals only. Peripheral $\mathrm{GFP}^{+}$cells still showed morphological signs of activation in the DHi (arrowhead), which was not observed in GFP>WT animals. (H) Stereological analysis of the lumbar spinal cord sections shown in $\mathbf{G}$ revealed a significantly higher number of GFP+ engrafted myeloid cells in the $\mathrm{DHi}$ in repopulated GFP $>$ TK mice $(n=8)$, but not in their GFP $>$ WT littermates $(n=9)$. (I and J) A significantly higher number of GFP+ engrafted myeloid cells was observed in the $\mathrm{DHi}$ of long-term repopulated mice ( $n=5$ /genotype). Scale bar: $500 \mu \mathrm{m}$. Inset images (original magnification, $\times 40$ ) are of the DHi. Error bars indicate the SEM. ${ }^{*} P<0.05,{ }^{* *} P<0.01$, and ${ }^{* *} P<0.001$, by paired, 2 -tailed Student's $t$ test for corresponding $\mathrm{DHc}$ and DHi pairs, except for the data in $\mathbf{F}$, for which a 1-way ANOVA with Bonferroni's post hoc analysis was used. Green double-asterisks in $\mathbf{H}$ and $\mathbf{J}$ indicate the difference between the GFP+ cells.

Importantly, however, we found that Calca, encoding the neuron-specific CGRP $\alpha$ protein that was shown to directly contribute to heat sensation $(8,17)$, was the one gene whose mRNA expression was significantly downregulated in the DH of the spinal cord in GFP > TK mice 7 dpi (Supplemental Table 1 and Figure 7A), while the expression levels of other neuronal transcripts, e.g., Cacna1b, Ntrk1, and Grin1, were unchanged (data not shown). To determine whether this change was detectable at the protein level, we also performed morphometric analysis of CGRP-IR in the $\mathrm{DH}$ of GFP>WT and GFP>TK animals. Independent of PSNL, we detected significantly lower expression levels of CGRP in microglia-depleted and myeloid cell-repopulated mice (Figure 7, $\mathrm{B}$ and C). Importantly, we found no reduction in the total neuronal population (Figure 7D), thus excluding the possibility that the loss of DH neurons caused the decrease in CGRP expression. To directly assess whether changes in neuronal CGRP expression can, in principle, be mediated by myeloid cells, we treated dorsal root ganglia (DRG) F11 cells with conditioned media from ATPand substance P-stimulated microglia and peritoneal macrophages and measured the release of CGRP from neurons. In line with our in vivo observations, we noticed a significant decrease in CGRP levels when neurons were subjected to conditioned media from myeloid cells (Figure 7E).

\section{Discussion}

As described for other models of neuropathic pain, we found that myeloid cells, including both resident microglia and peripherally derived myeloid cells, were activated and responded to the nerve injury in a temporally and spatially restricted fashion within the lumbar spinal cord, indicating that resident microglia and/or peripheral myeloid cells can influence the initiation of persistent pain in WT mice $(1,2,12,18)$. Previous findings point to an involvement of myeloid cells in the initiation of exaggerated pain responses, such as attenuated mechanical allodynia in rats after spinal nerve transection upon nonselective inhibition of spinal cord myeloid cells by minocycline (19). In addition, mice deficient in the chemokine receptor CCR2 or the fractalkine receptor CX3CR1 failed to develop mechanical allodynia and/or thermal hyperalgesia after PSNL $(20,21)$, while mice with a microglia-specific lack of brain-derived neurotrophic factor (BDNF) failed to develop morphine-induced hyperalgesia (22). These findings support the hypothesis that myeloid cells modulate neuronal activity in the spinal cord following peripheral nerve injury. However, methodological limitations of the approaches utilized thus far left many unanswered questions. For example, minocycline, besides conferring numerous divergent effects apart from antiinflammation $(23,24)$, has been shown to be insufficient in preventing aspects of microglia activation, particularly after nerve injury (25). Furthermore, genetic deletion of microglia-specific receptors and signaling pathways may lead to abnormal compensatory changes in mutant microglia, thus confounding data interpretation. Therefore, we set out to dissect the precise role of resident microglia by using male CD11b-HSVTK-transgenic mice (4) to specifically and entirely deplete these cells in a neuropathic pain setting. Male animals were used to avoid confounders such as the timing of ovulation.

Unlike other microglia depletion models in which ablated microglia are replaced in the CNS intrinsically through the proliferation of endogenous microglia (26), depletion of microglia in TK mice has been shown to induce rapid recruitment of peripheral myeloid cells into the brain parenchyma upon i.c.v. administration of GCV $(6,7)$, making this model uniquely suited to studying the differential effects of microglia and peripheral myeloid cells in the brain. Thus, TK mice differ from other published microglia depletion models, since in the latter, microglia are replaced by proliferating cells within the CNS, most likely because of a difference in the molecular mechanism of depletion $(26,27)$. Therefore, we examined neuropathic pain and related mechanisms in the absence of resident microglia and presence of peripheral myeloid cells to identify shared myeloid contributions to the neuropathic pain phenotype and uncover behavioral distinctions between CNS-resident and peripheral myeloid cells in this setting.

Surprisingly, infiltrating myeloid cells showed activation in the DHi up to 50 days after PSNL, when the activation of resident microglia has usually subsided, suggesting a differential response of endogenous microglia and peripherally derived CNS myeloid cells to PSNL at late time points. Moreover, microglia-depleted GFP $>$ TK animals harboring peripherally derived myeloid cells had considerably decreased PWTs in response to mechanical or cold, but not heat, stimuli ipsilateral to the PSNL at all of the time points tested, indicating a selective role of microglia in the etiology of PSNL-induced heat sensitivity, while in other pain modalities namely mechanical or cold sensation - peripheral myeloid cells were able to take over functions of local microglia. However, mechanistically, we cannot rule out the possibility that the observed lack of heat hyperalgesia is a consequence of pain-modulating mediators that are released by the high numbers of infiltrating peripheral myeloid cells, conversely implicating peripheral myeloid cells as unique modulators of pain. The finding that microglia deple- 
A

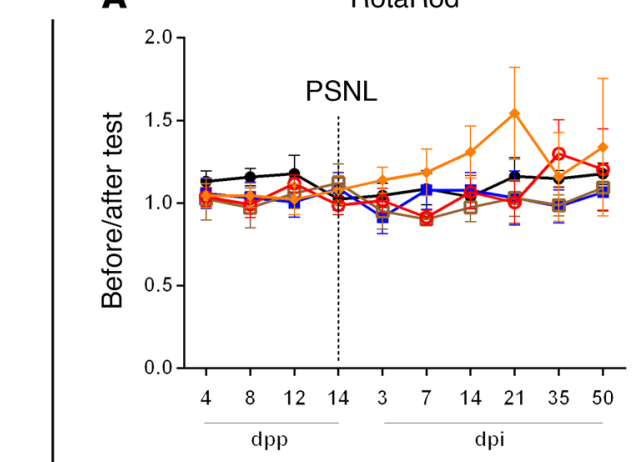

C

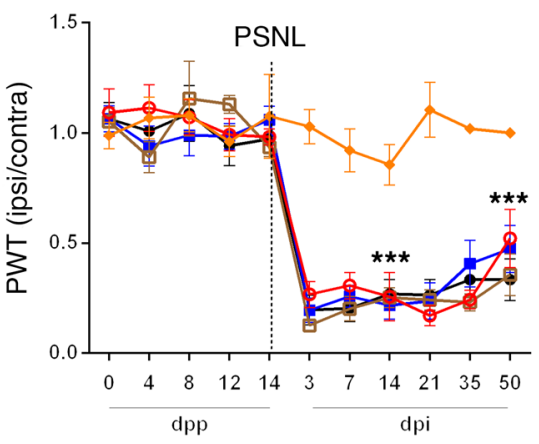

E

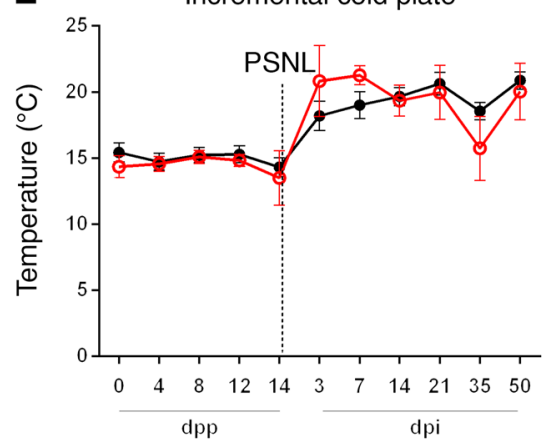

$\mathbf{F}$

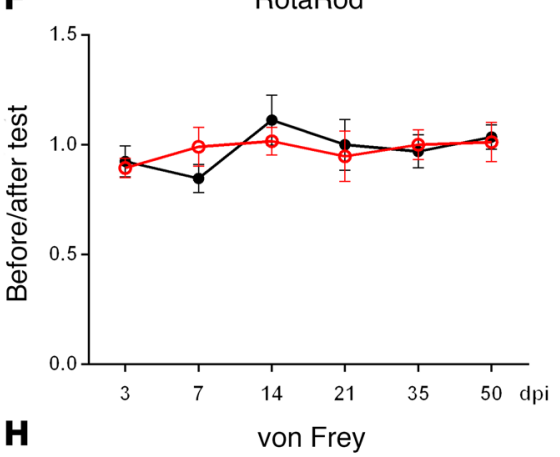

H

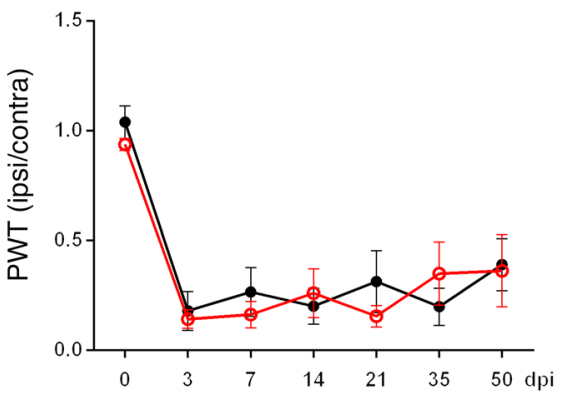

B

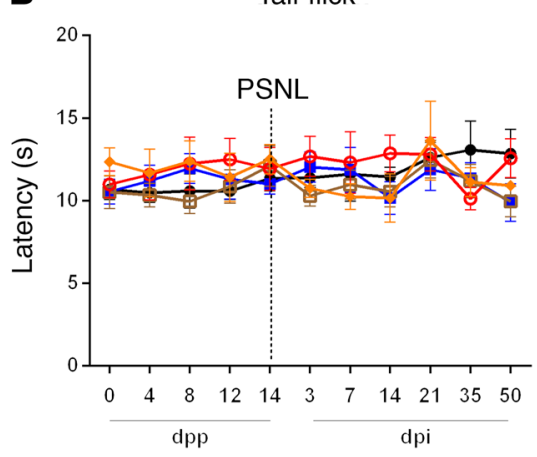

D

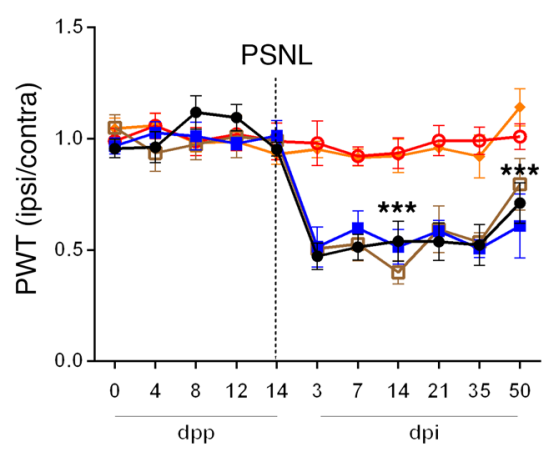

G

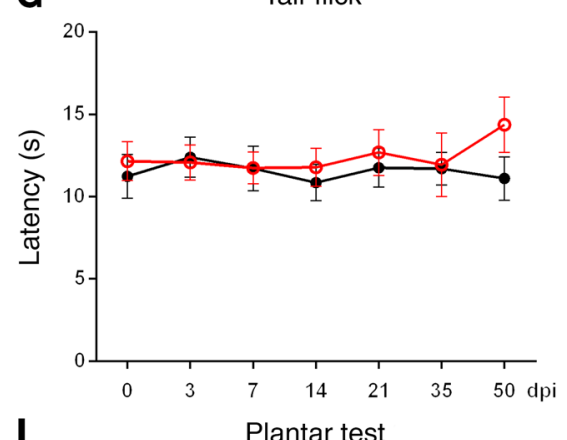

I

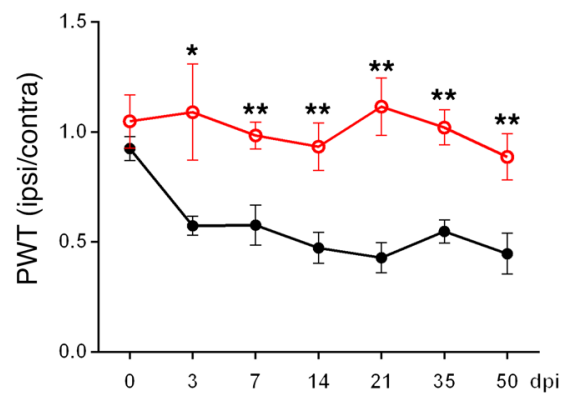

Figure 5. Microglia-depleted mice lack heat hyperalgesia. (A and $\mathbf{B}$ ) No deficits in the RotaRod or tail-flick test were observed in GCV- or aCSF-treated GFP $>$ WT or GFP $>$ TK animals, or in sham-operated GFP $>$ TK controls several days post pump (dpp) implantation, up to 50 days after PSNL. (C and $\mathbf{D})$ No changes in baseline values were observed before PSNL. Mechanical allodynia and heat hyperalgesia were detectable in GCV-treated GFP $>$ WT $(n=8)$, aCSF-treated GFP $>$ WT $(n=8)$, and aCSF-treated GFP $>$ TK $(n=8)$ animals after PSNL. In GCV-treated GFP > TK mice ( $n=8$ up to $21 \mathrm{dpi}, n=6$ for 35 and $50 \mathrm{dpi}$ ), PSNL resulted in a lasting formation of mechanical allodynia, but not heat hyperalgesia. Sham-operated GFP $>$ TK mice ( $n=8$ up to $7 \mathrm{dpi}, n=5-6$ for 14 and $21 \mathrm{dpi}, n=2-4$ for 35 and $50 \mathrm{dpi}$ ) developed neither mechanical allodynia nor heat hyperalgesia. (E) Increased sensitivity to cold stimuli was only detected after PSNL in GFP >WT $(n=9)$ and GFP $>$ TK $(n=7$ up to $8 \mathrm{dpp}, n=4-6$ from $12 \mathrm{dpp}$ onward) mice. ( $\mathbf{F}$ and $\mathbf{G}$ ) At long-term repopulation time points, no deficits in the RotaRod or tail-flick test were observed in GFP $>$ WT or GFP > TK mice. ( $H$ and I) GCV-treated, longterm repopulated GFP $>$ TK mice $(n=5 /$ genotype) lacked heat hyperalgesia, but developed lasting mechanical allodynia. Error bars indicate the SEM. Linear mixed models with adjustment for multiple testing were used for statistical analysis. In post hoc tests, group differences on days 14 and 50 after PSNL were tested. Adjustment for multiple testing was done within each model using Bonferroni's correction (A-D). Significant differences were determined for GCV-treated GFP>WT, aCSF-treated GFP $>W T$, aCSF-treated GFP $>$ TK, and GCV-treated GFP $>$ TK mice versus sham-treated mice (C and $\mathbf{D}) .{ }^{*} P<0.05$, ${ }^{* *} P<0.01$, and ${ }^{* *} P<0.001$, by paired, 2-tailed Student's $t$ test (E-I). 

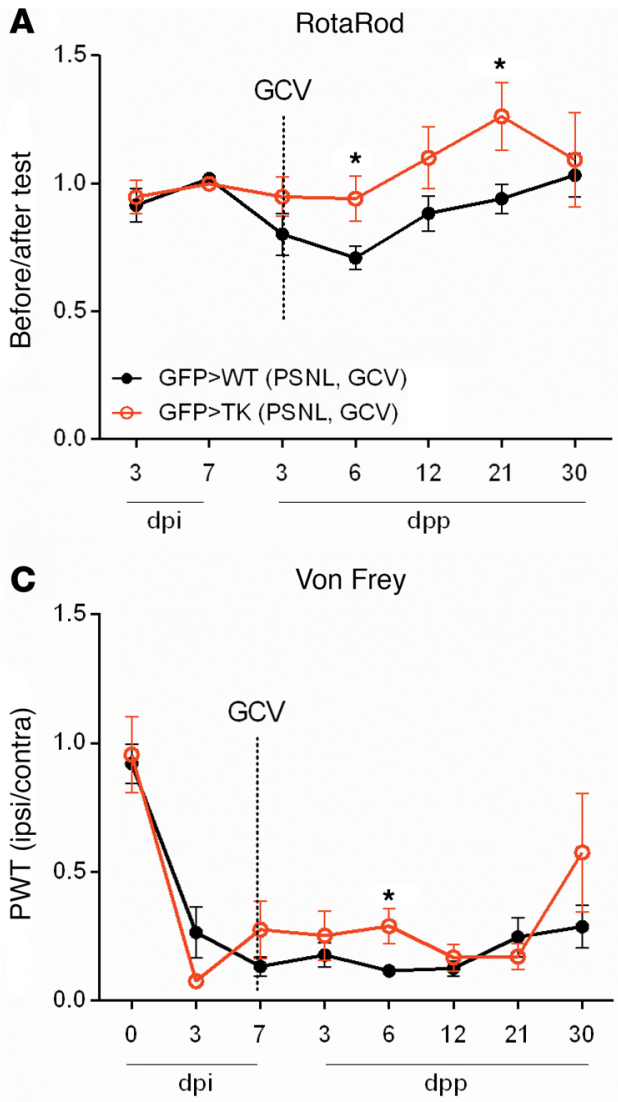

B

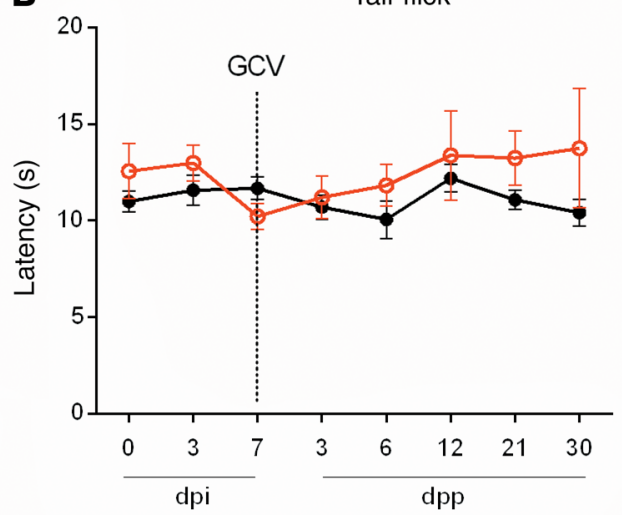

D

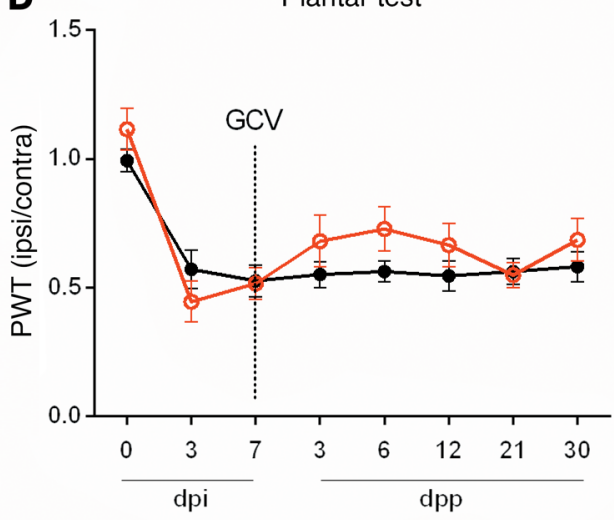

Figure 6. No effect of post-injury microglia depletion and myeloid cell repopulation on existing heat hyperalgesia and mechanical allodynia. (A) Comparable RotaRod performances were observed between GCV-treated GFP $>$ TK and GFP $>$ WT mice, with the exception of improved performance on days 6 and 21 after pump implantation. (B) GFP >WT and GFP > TK mice showed no deficits in tail-flick tests. (C and $\mathbf{D}$ ) GFP $>$ WT $(n=7)$ and GFP $>$ TK $(n=5$, $n=3-4$ for 21 and $30 \mathrm{dpp}$ ) mice developed mechanical allodynia and thermal hyperalgesia after PSNL. These neuropathic pain symptoms were not influenced by microglia depletion or myeloid cell repopulation in either genotype. Error bars indicate the SEM. ${ }^{*} P<0.05$, by paired, 2-tailed Student's $t$ test. tion and myeloid cell repopulation do not influence established chronic pain symptoms is in line with the theory that microglia are involved in early and acute responses to nerve injury, but not for the sustainment of neuropathic pain symptoms, while astrocytes are the main players in the maintenance of neuropathic pain, but not critically involved in its development $(28,29)$. Microglia have been shown to participate in central sensitization by modulation of synaptic transmission and amplification of pronociceptive signals, for example, via the release of TNF- $\alpha$, IL- 6 , and BDNF in the DH of the spinal cord $(30,31)$. When we examined the transcription pattern of resident microglia and engrafted myeloid cells, none of the above-mentioned genes was differentially expressed between GFP $>$ WT and GFP $>$ TK mice. However, we identified a number of presumably microglia-/macrophage-specific inflammatory markers, such as Cdl12 and Ccr2, that were significantly upregulated in repopulated GFP $>$ TK mice. Since the identified genes were specific to the condition of microglia depletion and myeloid cell repopulation, but not related to the PSNL, they appear to regulate the process of chemokine-mediated recruitment of peripheral myeloid cells to the CNS. This is in line with recent studies in the field demonstrating that, indeed, microglia do express a genetic profile distinct from that of peripheral myeloid cells (32-34).

When further dissecting the molecular underpinnings of how resident microglia specifically and distinctively mediate thermal hyperalgesia, we only found Calca (the gene encoding CGRP $\alpha$ ), among 84 genes, to be substantially downregulated in the DH of the lumbar spinal cord of GFP > TK mice. While our in vitro experiment further supports the notion that myeloid cells act as critical regulators of CGRP expression or survival of CGRP-expressing somatosensory neurons, it does not suffice to distinguish defined in vivo functions of resident microglia from those of peripheral myeloid cells, since myeloid cells in vitro are known to adopt myriad phenotypes $(32,35)$ that contrast with the in vivo setting, in which the CNS microenvironment orchestrates and confines microglia phenotypes. For this reason, the experimental in vivo setting described here was necessary to reliably address the question of whether myeloid cells of various origins indeed execute distinct functions independently of their microenvironment.

Notably, heat hyperalgesia, in particular, is mediated by antinociceptive CGRP expression (17). Importantly, CGRP-IR of DRG neurons was shown to directly contribute to noxious heat sensation, while mechanosensation was unaffected in CGRP $\alpha-$ $\mathrm{DTR}^{+/-}$mice (8), thus providing an explanation for the phenotype of GFP $>$ TK animals described in the present study. While we found diminished CGRP expression on both the injured and uninjured sides of the spinal DH (as a consequence of the repopulation process and not the PSNL), we did not detect increased heat withdrawal latencies in the contralateral paw. This suggests that a broader loss of CGRP $\alpha$-lineage neurons is required for a complete lack of heat sensitivity in the uninjured paw (8), whereas a mild reduction in CGRP expression appears to be sufficient to result in a loss of heat hypersensitivity under pathological conditions, such as PSNL, thus introducing a novel, non-neuronal approach to manipulating pathological pain. Because homeostatic and activated microglia also interact with and thereby shape the connectivity and function of the tripartite synapse in the healthy CNS (36-38), compromised synaptic transmission by $\mathrm{CGRP}^{+}$primary sensory neurons is conceivable in the microglia-depleted 


\section{Table 1. Experimental groups depicting all experimental procedures}

\begin{tabular}{|c|c|c|c|c|c|c|}
\hline Figure & Mice & BMCs & i.c.v. treatment & PSNL & After PSNL & After first manipulation \\
\hline $1, A-F$ & WT & - & - & Yes or sham & 2-90 dpi & $0-13$ wk \\
\hline $1 \mathrm{G}$ & WT & - & - & Yes & 7 dpi & 9 wk \\
\hline $2 \mathrm{~A}$ & TK & - & GCV & Yes & $50 \mathrm{dpi}$ & 17 wk \\
\hline $2 B$ & WT and TK & - & GCV & - & - & 10 wk \\
\hline $2 \mathrm{C}, 4, \mathrm{G}$ and $\mathrm{H}$ & WT and TK & - & $\mathrm{GCV}$ & Yes & $50 \mathrm{dpi}$ & 17 wk \\
\hline $2 \mathrm{D}, 4 \mathrm{C}$ & WT and TK & - & $\mathrm{aCSF}$ & Yes & 50 dpi & 17 wk \\
\hline $2 \mathrm{E}, 4 \mathrm{~B}$ & WT and TK & - & - & - & - & 8 wk \\
\hline $3, A$ and $B, 4, I$ and $J, 5, F-I$ & WT and TK & - & $\mathrm{GCV}$ & yes & $50 \mathrm{dpi}$ & 31 wk \\
\hline $3 C$ & WT and TK & - & GCV & Yes & $50 \mathrm{dpi}$ & 17 and 31 wk \\
\hline $4 \mathrm{~A}, 7, \mathrm{~A}-\mathrm{E}$ & WT and TK & - & $\mathrm{GCV}$ & Yes & 7 dpi & 11 wk \\
\hline $4, D$ and $E$ & WT & - & GCV & Sham & $50 \mathrm{dpi}$ & 17 wk \\
\hline $4 \mathrm{~F}$ & WT and TK & - & GCV or aCSF & Yes & 50 dpi & 17 and 31 wk \\
\hline $5, A-E$ & WT and TK & - & GCV or aCSF & Yes or sham & $50 \mathrm{dpi}$ & 17 wk \\
\hline $6, A-D$ & WT and TK & - & GCV & Yes & 37 dpi & $13 w k$ \\
\hline
\end{tabular}

BMCs, bone marrow chimeras.

and myeloid cell-repopulated setting, given the close proximity of activated microglia and peripheral myeloid cells with the central terminal zone of the injured afferents (39-41). However, we did not detect a differential expression of Calca or CGRP-IR within DRG between GFP $>$ WT and GFP $>$ TK mice (data not shown). This might be explained by the fact that i.c.v. GCV treatment does not deplete Iba1 ${ }^{+}$satellite glia outside the CNS and therefore does not trigger a reduction in CGRP expression. Moreover, CGRP is known to be locally synthesized in the spinal cord, e.g., in deeper lamina DH neurons (42), as well as during axonal regeneration (43), indicating that microglia depletion and/or myeloid cell repopulation in the $\mathrm{DH}$ directly target this local CGRP synthesis via noncontact-mediated effects that could be solely responsible for or act in conjunction with contact-mediated processes such as synaptic pruning.

Taken together, our data show that peripheral nerve injuryinduced microglia activation in the $\mathrm{DH}$ of the spinal cord plays a critical and unique regulatory role that serves to modulate the activity of CGRP $\alpha$ primary sensory neurons, a function that infiltrating myeloid cells cannot necessarily incur. Importantly, the overall physiological potential of these 2 cell populations, namely, resident microglia and peripherally derived myeloid cells, can be considered both distinct and redundant, depending on the pain entity. Experimental approaches in similar preclinical animal models involving the introduction of genetically engineered myeloid cells to the CNS following depletion of resident microglia upon PSNL may serve to elucidate the molecular underpinnings of microglia-neuronal crosstalk in the context of neuropathic pain. This knowledge may ultimately allow for the direct modification of CNS myeloid cell actions to modulate pathologic pain responses and lead to the development of novel, targeted, cell-based therapeutic intervention.

\section{Methods}

Animals. All experiments were conducted using 150- to 200-dayold adult male hemizygous CD11b-HSVTK (TK) mice (4) or their transgene-negative littermates (referred to as WT mice), which were originally derived from a B6D2 background and backcrossed with C57BL/6 mice for more than 12 generations. Mice were kept under pathogen-free and temperature- and humidity-controlled conditions, on a 12-hour light/12-hour dark cycle, with ad libitum access to food and water. Data from all animals were included for all respective analyses unless an animal had to be excluded because of death prior to completion of the study.

In vivo manipulations. Bone marrow chimeric mice were generated as described previously (5). Briefly, recipient mice were exposed to 10 Gy whole-body irradiation, after which they received an i.v. injection of $1 \times 10^{7}$ bone marrow cells obtained from the tibiae and femurs of B6-Tg(ACTbEGFP)1Osb mice (The Jackson Laboratory). Subsequently, mice received treatment with $0.01 \%$ enrofloxacin antibiotics (Baytril, Bayer Vital) for 1 month. The animals were subjected to PSNL 10 weeks (short term) or 6 months (long term) after transplantation. For i.c.v. GCV (8 mg/ml Cymevene, Roche) treatment, a mini-osmotic pump (Model 2002, Alzet, $0.5 \mu \mathrm{l} / \mathrm{h}$ ) was implanted as previously described (5). After 4 weeks, the pump reservoir was removed without disturbing the brain infusion cannula. Table 1 lists the various experimental groups.

Peripheral nerve injury. PSNL was conducted under ketamine/ xylazine (ketamine $100 \mathrm{mg} / \mathrm{kg}$, xylazine $10 \mathrm{mg} / \mathrm{kg}$ ) anesthesia. The right sciatic nerve was exposed at high thigh level, and the dorsal onehalf to one-third diameter of the common sciatic nerve was tightly ligated with 10-0 Prolene silk (Ethicon). In sham-operated animals, the nerve was exposed as in the PSNL procedure, but not ligated.

Antibodies. The following antibodies were used: rabbit anti-Iba1 (Wako Chemicals, 019-19741, 1:500 dilution); rabbit anti-GFP (Abcam, ab290, 1:1,000 dilution); rat anti-BrdU (AbD Serotec, OBT0030G, 1:500 dilution); mouse anti-NeuN (Millipore, MAB377, 1:500 dilution); and rabbit anti-CGRP (Enzo Life Sciences, CA1137, 1:200 dilution).

Histology and confocal microscopy. Animals were perfused with isotonic $\mathrm{NaCl}(0.9 \%)$ solution, followed by $4 \%$ paraformaldehyde (PFA) under deep ketamine/xylazine anesthesia (ketamine $100 \mathrm{mg} /$ $\mathrm{kg}$; xylazine $10 \mathrm{mg} / \mathrm{kg}$ ). Spinal cords were removed and fixed in $4 \%$ PFA for 2 days. Subsequently, L4-L6 segments were removed and immersed in $30 \%$ sucrose for at least 24 hours at $4^{\circ} \mathrm{C}$. Coronal sections (30- $\mu \mathrm{m}$-thick) were cut on a cryostat and processed free-floating. Sec- 
A

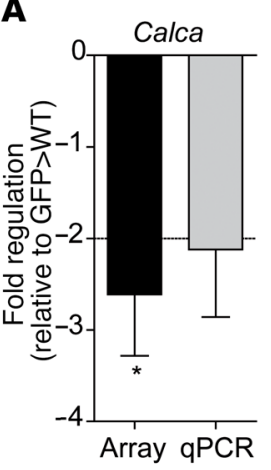

C

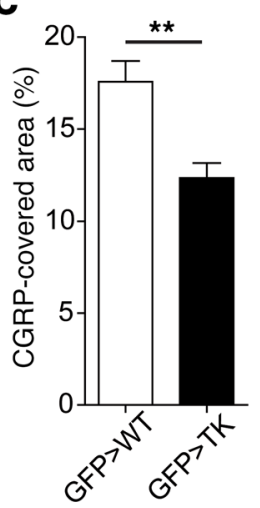

B

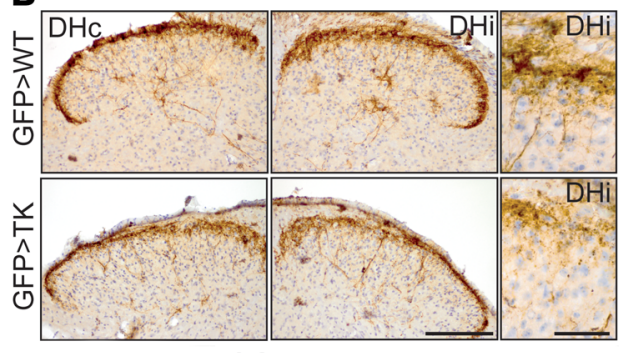

CGRP-IR; 7 dpi

D

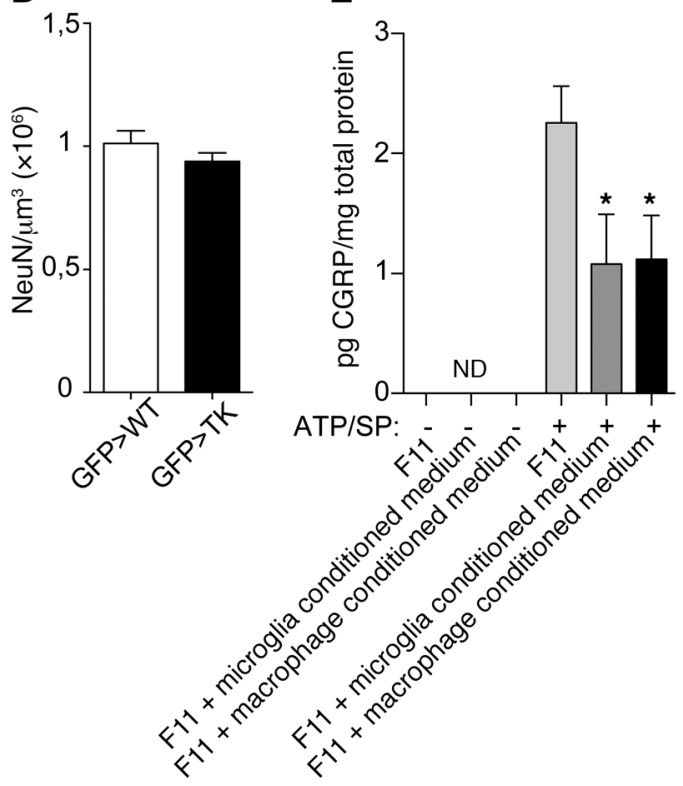

Figure 7. Reduced CGRP expression in the dorsal spinal cord of GFP > TK animals. (A) Differentially expressed Calca in GFP $>$ TK mice relative to GFP $>$ WT mice in array $(n=4$ / genotype) and by qPCR ( $n=3-4 /$ genotype) analyses. Data were normalized to Gapdh levels. (B) Central termination of sensory neurons. Representative images of CGRP-IR in cross sections of the lumbar dorsal spinal cord $7 \mathrm{dpi}$ in GFP >WT and GFP > TK mice. Scale bars: $500 \mu \mathrm{m}$ and $50 \mu \mathrm{m}$ (insets). (C) Quantitative morphometric analysis of lamina I of the $\mathrm{DH}$ revealed a significantly lower CGRP-covered area in GFP>TK $(n=5)$ mice than in GFP $>$ WT $(n=6)$ littermates. (D) Quantification of the number of $\mathrm{NeuN}^{+}$neurons within the lumbar spinal cord of GFP $>$ WT $(n=6)$ and GFP $>$ TK $(n=4)$ mice revealed no differences. (E) F11 DRG cells treated with conditioned medium derived from microglia or from peritoneal macrophages (technical triplicates) showed reduced CGRP protein release compared with those that were treated with fresh medium alone. In the absence of ATP and substance P (SP), CGRP was not detectable (ND). $C M$, conditioned medium. Error bars indicate the SEM. ${ }^{*} P<0.05$ and ${ }^{* *} P<0.01$, by paired, 2-tailed Student's $t$ test (A, C, and $\mathbf{D})$ and 1-way ANOVA with Bonferroni's post hoc analysis (E). tions were incubated for 1 hour at room temperature in PBS containing $0.3 \%$ Triton X-100 and 10\% normal goat serum (NGS). Primary antibodies were applied and incubated overnight at $4^{\circ} \mathrm{C}$ in PBS containing $0.3 \%$ Triton X-100 and 5\% NGS. After 3 washes in PBS, spinal sections were incubated with secondary antibodies conjugated to Alexa Fluor or avidin-peroxidase (Dianova, 1:300 dilution). For BrdU labeling, sections were pretreated with $50 \%$ formamide in $2 \times$ SSC for 2 hours at $65^{\circ} \mathrm{C}$, followed by $2 \times \mathrm{SSC}$ for 5 minutes twice at room temperature, 30 minutes in $2 \mathrm{~N} \mathrm{HCl}$ at $37^{\circ} \mathrm{C}$, and 10 minutes in $0.1 \mathrm{M}$ borate buffer prior to immunostaining. Multiple immunofluorescence images were acquired using a Zeiss LSM 5 Exciter confocal laser-scanning system. Images were processed in Adobe Photoshop CS3.

Cell counts and quantification. Morphometric quantification of reactive microglia in WT mice after PSNL was performed using the Cell D Analysis System, version 5.1 (Olympus). Defined regions containing the DHi and DHc in the L4-L6 spinal cord of 8 to 10 randomly selected sections per animal ( $n=4 /$ time point) stained with Iba1 were analyzed by determining the percentage of area covered by $\mathrm{Iba}^{+}$cells, using the color filter and phase analysis tool. The total number of BrdU-labeled cells was quantified in the DHi and DHc in the L4-L6 spinal cord (8-10 sections/animal, $n=4$ ) using a light microscope. For the quantification of double-positive BrdU and Iba1 cells, confocal $Z$-stacks were generated to confirm signal colocalization. Two sections per animal $(n=5)$ at 2 or
4 dpi were scanned at $\times 20$ magnification for BrdU- and Iba1-colabeled cells. For reconstituted mice, the number of microglia and peripherally derived myeloid cells was stereologically assessed in the lumbar spinal cord on random sets of every tenth systematically sampled $30-\mu \mathrm{m}$-thick Iba1- and GFP-immunostained section (yielding 10-12 sections/ mouse). Analysis was performed with StereoInvestigator software, version 10 (MBF Bioscience) and a motorized $x-y-z$ stage coupled to a video microscopy system (Olympus BX35F) using the optical fractionator workflow (44). NeuN ${ }^{+}$neuronal cell bodies were quantified throughout the entire $\mathrm{DH}$, represented by 12 systematically sampled $30-\mu \mathrm{m}$-thick free-floating sections per animal. Analyses were performed with the aid of StereoInvestigator software, version 10, and a motorized $x-y-z$ stage coupled to a video microscopy system (Olympus BX35F) using the meander scan function. Measurements of the distance between neighboring cell bodies and cross-sectional areas of $\mathrm{Iba1}^{+}$microglia (20 distances or cell bodies/section, 5 sections/animal, $n>4$ /group) were made using the CellSense, version 1.6 (Olympus), analysis system for mean distance and cell body size analyses, respectively. We performed morphometric analysis of the immunohistochemical $\mathrm{CGRP}^{+}$area of i.c.v. GCV-treated GFP $>$ WT and GFP $>$ TK mice using the CellSense analysis system in 6 to 7 sections of the lumbar DH.

Behavioral studies. As a prerequisite for inclusion in the behavioral data analysis, we confirmed the efficacy of microglia depletion 
and repopulation (>70\%) by assessment of the lumbar spinal cord of all GFP > TK mice. Before behavioral testing, mice were acclimated to the testing room, the equipment, and the experimenter for 1 week. To evaluate the effects of microglia depletion on thermal hyperalgesia and mechanical allodynia and motor function and reflexes, mice underwent a comprehensive behavioral test battery before surgery and $4,8,12$, and 14 days after i.c.v. treatment, as well as 3, 7, 14, 21, 35 , and 50 dpi. Mice were acclimated to all testing procedures for 10 minutes each for 1 week prior to the start of the experiment, for $5 \mathrm{~min}$ utes before each testing session, and for 10 minutes each on a daily basis throughout the entire testing period. For the von Frey test, punctate tactile sensitivity was measured according to threshold responses to calibrated retractable von Frey monofilaments (BioSeb), based on the up-down method in rats (45). Depending on the response of the animal, increasing or decreasing strengths of von Frey filaments were applied sequentially to the mid-line of the plantar surface of each hind paw. The stimulus intensity threshold represents the smallest force that repeatedly elicits withdrawal of the hind paw during 9 trials. Together with the force of the final filament, the $50 \%$ response threshold was calculated for both ipsilateral and contralateral paws and expressed as ipsilateral/contralateral ratios. For the tail-flick test, the tails of mice were exposed to a radiant heat source (25\%) until tail withdrawal (flicking response) or signs of struggle. A cut-off time of 30 seconds was imposed to avoid injury to the tail. For the plantar test, thermal sensitivity was tested using the Hargreaves method (46) with a plantar test apparatus (IITC Life Sciences, Series 8, Model $390)$. The latency to withdraw the ipsilateral and contralateral hind paws from the light radiant heat source (25\%) placed underneath was recorded. Both hind paws were tested twice, with at least 5 minutes in between. To avoid tissue damage, the heat stimulus was removed after 30 seconds. Means were calculated and expressed as ipsilateral/ contralateral ratios. For the dynamic cold plate test, cold hyperalgesia was assessed on the incremental cold plate (IITC Life Sciences, Series 8, Model PE34). A cut-off temperature of $0^{\circ} \mathrm{C}$ was imposed to avoid potential tissue damage. Mice were immediately removed from the cold plate after a reaction (paw licking, flinching, or shaking) was observed. To avoid cold stress, 2 test rounds were performed with an interval of 1 hour. The RotaRod test is a standard test for motor function, coordination, and balance. After several training sessions (three 10-rpm trials and two 20-rpm trials over two days each), mice were tested on the RotaRod (TSE Systems, Series 3375), which increased in speed from $4 \mathrm{rpm}$ to $40 \mathrm{rpm}$ within 300 seconds. The latency to fall off the rod was measured in 2 consecutive trials, and the mean latency was used for analysis.

RNA extraction. L4-L6 tissue was vertically separated along the median and further dissected to the DHi and DHc. Tissue pieces were subjected to total RNA extraction using the InviTrap Spin Tissue RNA Mini Kit (Invitek Inc.). Quality and ribosomal RNA band integrity of isolated RNA was assessed by determining the integrity of the 28S and $18 \mathrm{~S}$ bands using the Agilent 6000 Nano Kit (Agilent Technologies) and measured with the Bioanalyzer 2100 (Agilent Technologies) according to the manufacturer's instructions.

Quantitative real-time PCR array. For the PCR array-based analysis of pain-related genes, 200 ng RNA obtained from the DHi and DHc of GFP $>$ WT and GFP $>$ TK mice ( $n=4$ /group) was reverse transcribed using the RT ${ }^{2}$ First Strand Kit (SABiosciences, QIAGEN) according to the manufacturer's instructions. The $\mathrm{RT}^{2}$ Profiler PCR Array PAMM-
162ZA (SABiosciences, QIAGEN) was used for gene expression analysis. All steps were performed according to the manufacturer's protocol for the ABI 7900HT Sequence Detection System. The specificity of amplification was assessed by melting curve evaluation. Webbased analysis of the 84 genes was performed on the company's website (http://www.sabiosciences.com/pcrarraydataanalysis.php). Data were normalized to Gapdh.

Complementary DNA synthesis and quantitative real-time PCR. RNA (600 ng) was converted to cDNA using the QuantiTect Reverse Transcription Kit (QIAGEN), according to the manufacturer's protocol. To detect mRNA expression in DH samples from GFP $>$ WT and GFP $>$ TK animals ( $n=4$ /group), quantitative real-time PCR was performed using on-demand TaqMan gene expression assays (Life Technologies, Thermo Fisher Scientific) for Calca (Mm03749347_m1) on the ABI 7900HT Real-Time PCR System. Samples were run in triplicate for each individual condition. The relative expression levels of the target gene were normalized to that of Gapdh. Subsequent analysis was performed using the $\Delta / \Delta$ Ct method.

Cell culture, FACS, and ELISA in vitro assays. To measure CGRP levels, DRG F11 cells (ECACC 08062601, provided by Robert Wellhausen, Fraunhofer IZI, Berlin, Germany) were treated with conditioned media derived from FACS-sorted microglia or from peritoneal macrophages that were subjected to $5 \mathrm{mM}$ ATP and $6.2 \mathrm{mM}$ substance $\mathrm{P}$ for 3 hours. After 1 hour, the supernatant was removed and stored at $-80^{\circ} \mathrm{C}$ until use. As a control, additional F11 cells were either treated with ATP and substance P or with medium only for 1 hour. CGRP concentration was determined using a CGRP-ELISA Kit (CEA876Mu, Cloud-Clone Corp.) according to the manufacturer's instructions. Each sample was run in triplicate, and CGRP concentrations were calculated against total protein content in the samples, which was assessed following the Pierce BCA Protein Assay instructions (Thermo Fisher Scientific).

Statistics. Statistical analysis was performed using SPSS software (IBM). For pairwise comparisons of experimental groups, a 2-tailed Student's $t$ test was used. To compare the mean of 3 or more samples, a 1-way ANOVA with Bonferroni's post hoc analysis was used. We applied linear mixed models (random intercept), which used all available data on all mice in 1 model for each outcome separately in Figure 5. In these models, the specific outcome was the dependent variable, while dummy codes for the group (GROUP) and the time range after the surgery (SURGERY, coded 1 for all time points after surgery and 0 before) as well as a covariate for time (TIME) (in days over the whole study period) were the independent variables. We additionally included interaction terms for GROUP $\times$ SURGERY, TIME $\times$ SURGERY, and TIME $\times$ GROUP) to account for differential changes in the groups. In post hoc tests, we tested group differences at 14 and 50 dpi. Adjustment for multiple testing was done within each model using Bonferroni's correction. Results are expressed as mean values \pm SEM. A $P$ value of less than 0.05 was considered statistically significant.

Study approval. All protocols were reviewed and approved by the IRB of the Landesamt für Gesundheit und Soziales (LAGeSo) (Berlin, Germany).

\section{Author contributions}

KRM and FLH designed the experiments. SK, REK, and CW conducted the experiments. SK and KRM wrote the manuscript, 
which was supervised and edited by FLH. MJ performed data analysis and edited the manuscript.

\section{Acknowledgments}

We thank Matthias Endres (Department of Neurology, Charite Universitätsmedizin Berlin) and York Winter (Animal Outcome Core Facility, Cluster of Excellence Neurocure, Charité - Universitätsmedizin Berlin) for allowing us to perform the behavioral tests in their units; Gerit Pfuhl (Animal Outcome Core Facility, Cluster of Excellence Neurocure, Charité - Universitätsmedizin Berlin) for instructing us on how to conduct the tests; Alexander Haake (Department of Neuropathology, Charité - Universitätsmedizin Berlin) for neuronal quantification; Claudia Hempt (Department of Neuropathology, Charité - Universitätsmedizin Berlin) and Sara Mirali (University of Toronto, Canada) for quantifying microglial mean distances and cell bodies; Robert Wellhausen (Fraunhofer Institute for Cell Therapy and Immunology IZI, Potsdam, Germany) for providing F11 cells; Helina Machelska and Christoph Stein (both from the Department of Anesthesiology and Critical Care Medicine, Charité - Universitätsmedizin Berlin, Germany) for discussions on the experimen- tal design and statistical analyses; and Ulrike Grittner (Institute of Biometry and Clinical Epidemiology, Charité - Universitätsmedizin Berlin) for biostatistical support in applying the linear mixed models. This work was supported by the Deutsche Forschungsgemeinschaft (SFB TRR 43, SFB TRR 167, NeuroCure Exc 257 and HE 3130/6-1, to FLH); the BIH (Collaborative Research Grant, to FLH); the DZNE - Berlin; and a fellowship from the ZIBI-IMPRS Graduate School (to SK).

Address correspondence to: Frank L. Heppner, Department of Neuropathology, Charitéplatz 1, D-10117 Berlin, Germany. Phone: 49.30.450.536042; Email: frank.heppner@charite.de.

SK's present address is: Apceth Biopharma GmbH, Munich, Germany.

KRM's present address is: NanoString Technologies, Seattle, Washington, USA.

RK's present address: Neurosurgical Research, University Clinics Munich, Ludwig-Maximilians-University, Munich, Germany.
1. Zhang J, De Koninck Y. Spatial and temporal relationship between monocyte chemoattractant protein-1 expression and spinal glial activation following peripheral nerve injury. J Neurochem. 2006;97(3):772-783.

2. Milligan ED, Watkins LR. Pathological and protective roles of glia in chronic pain. Nat Rev Neurosci. 2009;10(1):23-36.

3. Echeverry S, Shi XQ, Rivest S, Zhang J. Peripheral nerve injury alters blood-spinal cord barrier functional and molecular integrity through a selective inflammatory pathway. J Neurosci. 2011;31(30):10819-10828.

4. Heppner FL, et al. Experimental autoimmune encephalomyelitis repressed by microglial paralysis. Nat Med. 2005;11(2):146-152.

5. Grathwohl SA, et al. Formation and maintenance of Alzheimer's disease beta-amyloid plaques in the absence of microglia. Nat Neurosci. 2009;12(11):1361-1363.

6. Varvel NH, et al. Microglial repopulation model reveals a robust homeostatic process for replacing CNS myeloid cells. Proc Natl Acad Sci U S A. 2012;109(44):18150-18155.

7. Prokop S, et al. Impact of peripheral myeloid cells on amyloid- $\beta$ pathology in Alzheimer's diseaselike mice. JExp Med. 2015;212(11):1811-1818.

8. McCoy ES, Taylor-Blake B, Street SE, Pribisko AL, Zheng J, Zylka MJ. Peptidergic CGRP $\alpha$ primary sensory neurons encode heat and itch and tonically suppress sensitivity to cold. Neuron. 2013;78(1):138-151.

9. Colburn RW, Rickman AJ, DeLeo JA. The effect of site and type of nerve injury on spinal glial activation and neuropathic pain behavior. Exp Neurol. 1999;157(2):289-304.

10. Kettenmann H, Hanisch UK, Noda M, Verkhratsky A. Physiology of microglia. Physiol Rev. 2011;91(2):461-553.

11. Ginhoux F, et al. Fate mapping analysis reveals that adult microglia derive from primitive macro- phages. Science. 2010;330(6005):841-845

12. Zhang J, Shi XQ, Echeverry S, Mogil JS, De Koninck Y, Rivest S. Expression of CCR2 in both resident and bone marrow-derived microglia plays a critical role in neuropathic pain. J Neurosci. 2007;27(45):12396-12406.

13. Mildner A, et al. Microglia in the adult brain arise from Ly-6ChiCCR2+ monocytes only under defined host conditions. Nat Neurosci. 2007;10(12):1544-1553.

14. Sandkühler J. Models and mechanisms of hyperalgesia and allodynia. Physiol Rev. 2009;89(2):707-758.

15. Woolf CJ. Central sensitization: implications for the diagnosis and treatment of pain. Pain. 2011;152(3 Suppl):S2-15.

16. Basbaum AI, Bautista DM, Scherrer G, Julius D. Cellular and molecular mechanisms of pain. Cell. 2009;139(2):267-284.

17. Mogil JS, et al. Variable sensitivity to noxious heat is mediated by differential expression of the CGRP gene. Proc Natl Acad Sci U S A 2005;102(36):12938-12943.

18. McMahon SB, Malcangio M. Current challenges in glia-pain biology. Neuron. 2009;64(1):46-54.

19. Raghavendra V, Tanga F, DeLeo JA. Inhibition of microglial activation attenuates the development but not existing hypersensitivity in a rat model of neuropathy. J Pharmacol Exp Ther. 2003;306(2):624-630.

20. Abbadie C, et al. Impaired neuropathic pain responses in mice lacking the chemokine receptor CCR2. Proc Natl Acad Sci U S A. 2003;100(13):7947-7952.

21. Staniland AA, et al. Reduced inflammatory and neuropathic pain and decreased spinal microglial response in fractalkine receptor (CX3CR1) knockout mice. J Neurochem. 2010;114(4):1143-1157.

22. Ferrini F, et al. Morphine hyperalgesia gated through microglia-mediated disruption of neuronal $\mathrm{Cl}^{\prime}$ homeostasis. Nat Neurosci.
2013;16(2):183-192

23. Keller AF, Gravel M, Kriz J. Treatment with minocycline after disease onset alters astrocyte reactivity and increases microgliosis in SOD1 mutant mice. Exp Neurol. 2011;228(1):69-79.

24. Yang L, et al. Minocycline enhances MPTP toxicity to dopaminergic neurons. J Neurosci Res. 2003;74(2):278-285.

25. Fendrick SE, Miller KR, Streit WJ. Minocycline does not inhibit microglia proliferation or neuronal regeneration in the facial nucleus following crush injury. Neurosci Lett. 2005;385(3):220-223.

26. Elmore MR, et al. Colony-stimulating factor 1 receptor signaling is necessary for microglia viability, unmasking a microglia progenitor cell in the adult brain. Neuron. 2014;82(2):380-397.

27. Bruttger J, et al. Genetic Cell Ablation Reveals Clusters of Local Self-Renewing Microglia in the Mammalian Central Nervous System. Immunity. 2015;43(1):92-106.

28. Suter MR, Wen YR, Decosterd I, Ji RR. Do glial cells control pain? Neuron Glia Biol. 2007;3(3):255-268.

29. Vallejo R, Tilley DM, Vogel L, Benyamin R. The role of glia and the immune system in the development and maintenance of neuropathic pain. Pain Pract. 2010;10(3):167-184.

30. Kawasaki Y, Zhang L, Cheng JK, Ji RR. Cytokine mechanisms of central sensitization: distinct and overlapping role of interleukin-1beta, interleukin-6, and tumor necrosis factor-alpha in regulating synaptic and neuronal activity in the superficial spinal cord. JNeurosci. 2008;28(20):5189-5194.

31. Coull JA, et al. BDNF from microglia causes the shift in neuronal anion gradient underlying neuropathic pain. Nature. 2005;438(7070):1017-1021.

32. Butovsky $\mathrm{O}$, et al. Identification of a unique TGF- $\beta$-dependent molecular and functional signature in microglia. Nat Neurosci. 2014;17(1):131-143.

33. Buttgereit A, et al. Sall1 is a transcriptional regulator defining microglia identity and function. 
Nat Immunol. 2016;17(12):1397-1406.

34. Keren-Shaul H, et al. A Unique Microglia Type Associated with Restricting Development of Alzheimer's Disease. Cell. 2017;169(7): 1276-1290.e17.

35. Ransohoff RM. How neuroinflammation contributes to neurodegeneration. Science. 2016;353(6301):777-783.

36. Tremblay MÈ, Stevens B, Sierra A, Wake $\mathrm{H}, \mathrm{Bessis} \mathrm{A}$, Nimmerjahn A. The role of microglia in the healthy brain. J Neurosci. 2011;31(45):16064-16069.

37. Wake H, Moorhouse AJ, Jinno S, Kohsaka S, Nabekura J. Resting microglia directly monitor the functional state of synapses in vivo and determine the fate of ischemic terminals. J Neurosci. 2009;29(13):3974-3980.

38. Kettenmann H, Kirchhoff F, Verkhratsky A.
Microglia: new roles for the synaptic stripper. Neuron. 2013;77(1):10-18.

39. Liu L, Persson JK, Svensson M, Aldskogius H. Glial cell responses, complement, and clusterin in the central nervous system following dorsal root transection. Glia. 1998;23(3):221-238.

40. Winkelstein BA, Rutkowski MD, Sweitzer SM, Pahl JL, DeLeo JA. Nerve injury proximal or distal to the DRG induces similar spinal glial activation and selective cytokine expression but differential behavioral responses to pharmacologic treatment. J Comp Neurol. 2001;439(2):127-139.

41. Tsuda M, Inoue K, Salter MW. Neuropathic pain and spinal microglia: a big problem from molecules in "small" glia. Trends Neurosci. 2005;28(2):101-107.

42. Tie-Jun SS, Xu Z, Hökfelt T. The expression of calcitonin gene-related peptide in dorsal horn neurons of the mouse lumbar spinal cord. Neuroreport. 2001;12(4):739-743.

43. Toth CC, et al. Locally synthesized calcitonin gene-related Peptide has a critical role in peripheral nerve regeneration. J Neuropathol Exp Neurol. 2009;68(3):326-337.

44. Long JM, et al. Stereological estimation of total microglia number in mouse hippocampus. J Neurosci Methods. 1998;84(1-2):101-108.

45. Chaplan SR, Bach FW, Pogrel JW, Chung JM, Yaksh TL. Quantitative assessment of tactile allodynia in the rat paw. J Neurosci Methods. 1994;53(1):55-63.

46. Hargreaves K, Dubner R, Brown F, Flores C, Joris $\mathrm{J}$. A new and sensitive method for measuring thermal nociception in cutaneous hyperalgesia. Pain. 1988;32(1):77-88. 